\title{
Robust Catheter Tracking by Fusing Electromagnetic Tracking, Fiber Bragg Grating and Sparse Fluoroscopic Images
}

\author{
Xuan Thao Ha, Mouloud Ourak, Omar Al-Ahmad, Di Wu, Gianni Borghesan, \\ Arianna Menciassi, and Emmanuel Vander Poorten
}

\begin{abstract}
Endovascular catheterization is an intervention which offers a low risk alternative to open surgery in many patients. Today's interventions rely heavily on fluoroscopic imaging to guide interventionalists. Fluoroscopy only produces 2D visualization of the catheter and also exposes both the patient and interventionalists to harmful radiation. Different approaches have been proposed to overcome the limitations of fluoroscopy. Fiber Bragg Grating (FBG)-based shape sensing is becoming popular to reconstruct the catheter shape. Multi-core fibers with parallel optical cores are interesting as they allow 3D shape reconstruction with a single fiber. A common issue with FBG-based shape sensing is its sensitivity to variations in twist. Even small amounts of twist can significantly impact the overall shape reconstruction accuracy. This work proposes a novel approach which combines electromagnetic tracking (EMT), FBG-based shape sensing, and sparse fluoroscopic images. The method provides realtime 3D visualization of the catheter without the need of continuous fluoroscopy. A unique feature of the proposed method is the selective use of imaging for dynamic twist-compensation of the FBG sensor. The proposed sensor-fusion method improved 3D reconstruction accuracy. Real-world in-vitro experiments promising results. For a catheter with an embedded fiber length of $170 \mathrm{~mm}$, the proposed approach the 3D shape with a median root-mean-square (rms) error of $0.39 \mathrm{~mm}$ and an interquartile range of $0.10 \mathrm{~mm}$ in the $2 \mathrm{D}$ experiment in which the catheter was bent in a plane. A median rms error of $0.54 \mathrm{~mm}$ and an interquartile range of $0.07 \mathrm{~mm}$ were achieved in the $3 \mathrm{D}$ experiments.
\end{abstract}

Index Terms-Catheter, shape reconstruction, electromagnetic, Fiber Bragg Grating, dynamic twist.

\section{INTRODUCTION}

$\mathbf{C}$ ATHETER shape reconstruction has received considerable attention due to potential to offer guidance to interventionalists. Research in catheter shape estimation can be classified into three groups: model-based approaches, sensorbased approaches and the combination of the two, that we will refer as fusion approaches. The first group models the kinematic or dynamic behavior of catheters in different kinds

Manuscript received May 16, 2021; accepted August 14,2021. Date of publication August 20, 2021; date of current version August 20,2021. This work was supported by the ATLAS project. This project has received funding from the European Union's Horizon 2020 research and innovation program under the Marie Sklodowska-Curie grant agreement No 813782, and the internal KU Leuven C2 project SF-REACTA.

Xuan Thao $\mathrm{Ha}$ is with the Department of Mechanical Engineering, KU Leuven Univerisity 3000, Leuven, Belgium (email:xuanthao.ha@kuleuven.be).

Mouloud Ourak, Omar Al-Ahmad, Di Wu and Emmanuel Vander Poorten are with the Department of Mechanical Engineering, KU Leuven Univerisity 3000, Leuven, Belgium.

Gianni Borghesan is with Core Lab ROB, Flanders Make, Belgium and the Department of Mechanical Engineering, KU Leuven Univerisity 3000 , Leuven, Belgium.

Arianna Menciassi is with The BioRobotics Institute, Scuola Superiore Sant'Anna of Pisa 56025, Pontedera, Italy. of environments which may also be modeled. The performance of model-based approaches heavily relies on the accuracy of the modeling procedure. However, accurate models are highly complex and computationally intensive, which their intraoperative use., models are designed for specific anatomy and/or a specific instrument, thus limiting the applicability to other patients or instruments without undergoing serious additional modeling efforts. Different sensing modalities based on fluoroscopy, (EMT) or optical fibers have been proposed for catheter localization and shape sensing in the so-called sensorbased approaches. Unfortunately, each sensor modality suffers from drawbacks. Fluoroscopy only provides $2 \mathrm{D}$ visualization. This means that interventionalists have to correlate in their mind the 2D catheter with a 3D representation of the patient's anatomy. Biplanar fluoroscopy systems give interventionalists 2D images from two different angles. Wagner $e t$ al. proposed a method to reconstruct the 3D shape of an instrument during the procedure [1]. While this is an appealing method, radiation remains a problem. In fact, biplane imaging leads to higher radiation doses compared to monoplane fluoroscopy [2]. Several proposed to embed multiple EMT sensors along the catheter to track the position and to reconstruct the 
shape [3] [4]. The small size of EMT sensors allows their integration in flexible instruments with limited effect on the overall mechanical behavior of said instruments. However, EMT comes with some particular problems that complicate their adoption in clinical practice. Firstly, EMT systems do not offer uniform accuracy over the tracking volume. A second important disadvantage follows from their sensitivity to the presence of metallic or ferromagnetic materials. These materials will distort the electromagnetic field that is maintained by the EMT-generator. This distortion has a direct impact on the achievable estimation accuracy. Shape sensing based on optical fibers is becoming popular lately [5]-[8]. Intuitive (California, United States) developed a fiber optic based shape sensor technology to provide interventionalists with a precise shape of the catheter throughout the navigation during the bronchoscopy. However, FBGs suffer from spectral distortion upon bending. . The resulting integration errors can cause quite significant deviations [13]. A major challenge so far using FBGs in shape sensing has been that FBG fibers or multicore fibers cannot distinguish strain induced by twisting from strain induced by bending. To avoid this ambiguity, catheters are normally made from torsionally stiff elements to prevent torsion in the FBG. In practice, it is very difficult to design a catheter that protects the fiber completely from torsion. Given that even small amounts of twist have a large impact on the overall shape accuracy, the twist is considered one of the most important challenges to achieve precise shape reconstruction. Nevertheless, FBG-based shape sensing has been reported to have promising accuracy catheter shape sensing with an average error of $1.13 \mathrm{~mm}$ and maximum error of $2.11 \mathrm{~mm}$ in [5] and $0.70 \mathrm{~mm}$ and $2.52 \mathrm{~mm}$ in [7]. To measure these errors, the ground truth shape and the reconstructed shape were first aligned by iterative closest point algorithm [14]. Then, the errors were calculated by averaging distance between correspondent points of ground truth and reconstructed shape. However, in these works the absolute positioning, nor the error introduced by unmodeled twist has not been addressed, thus leaving space for improvement. Sensor fusion methods have been developed to overcome issues of pure model-based or pure sensor-based approaches. Dore et al. proposed a method to fuse electromagnetic tracking (EMT) with physically-based simulation [15]. The EMT sensor data and the estimated shape that was reconstructed by a catheter insertion model were combined to get a better shape estimation. The method from Dore et al. requires a pre-operative model of the patient's anatomy that needs to be aligned with the real patient's anatomy in a tedious registration procedure. Tran et al. fused the 3D shape from EMT sensors with fluoroscopic images [4]. By solving an optimization problem to maximize the correspondence between the $3 \mathrm{D}$ shape and the $2 \mathrm{D}$ shape in the image, the shape sensing accuracy was improved. In this approach, the initial shape reconstructed from the discrete EMT sensors. The nonuniformity in tracking accuracy of the EMT system affect the initial shape estimation and could lead to failure in solving the optimization problem., a large amount of EMT sensors are necessary to obtain a sufficiently accurate shape model. Overall, this makes the catheter complex, expensive and fragile. Azizian proposed to use a Kalman filter to fuse
EMT with X-ray images [16]. Alambeigi et al. introduced a stochastic algorithm to combine EMT and FBG to improve the tracking accuracy of unmodeled continuum manipulators [17]. While interesting Azizian's and Alambeigi's methods only focus on tracking the tip of the catheter where the entire shape is of interest in clinical applications. Zhang et al. proposed to fuse FBG shape sensing with stereo vision [18]. Although excellent accuracy was reported in Zhang's work (absolute position error of $0.67 \pm 0.07 \mathrm{~mm}$ ) stereo vision is typically not available in cardiovascular applications.

To overcome the shortcomings of the above-mentioned approaches, this work proposes a novel technique that makes use of EMT, FBG-based shape sensing and fluoroscopy. Compared to prior works, the proposed method allows drastically minimizing the use of fluoroscopy. The use of FBG sensors to estimate the catheter shape does not only help reduce safety problems arising from fluoroscopy imaging, it also provides a dense real-time 3D representation of the catheter shape, thus reducing the number of required embedded EMT sensors. In this work, only 2 EMT sensors are needed keeping the construction of the catheter fairly simple. The two EMT sensors and FBG fiber a limited effect on the overall stiffness of the catheter. By incorporating EMT sensors in the catheter, the reconstructed shape from FBG-based shape sensing can be localized in an external reference frame namely the EMT coordinate system. The combined EMT - FBG sensing could in theory suffice to estimate the full shape assuming that the shape provided by the FBGs is always correct, thus without error induced by twist. The main contribution of this work is exactly to solve the errors induced by catheter twist. A novel method that makes minimal use of fluoroscopy and fuses this with EMT and FBG is hetero developed. :

- a method for integrating a multi-core fiber and EMT sensors into a catheter and producing an absolute catheter shape expressed in the EMT coordinate system,

- an EMT - FBG co-registration method that derives the correspondence between the shape and the measured pose of the EMT sensors without the need of additional CT scan as suggested in [19],

- a method to fuse the FBG-based shape estimates with fluoroscopic images to estimate and compensate for the dynamic twist throughout the procedure.

This paper is organized as follows: Section II shows the design of our catheter followed by the proposed fusion framework which is described in Section III. Section IV outlines the setup that has been built to evaluate the accuracy of the proposed method. on 2D and 3D shapes for dynamic configurations with ground truth data are presented in Section IV. Section $\mathrm{V}$ concludes the paper summarizing the main results and sketching directions for future work.

\section{PLATFORM DESIGN}

To prove the feasibility of our approach, an experimental platform has been built as shown in Fig. 1. The setup consists of 4 modules: an interrogator (FBG-Scan 804, FBGS, Geel, Belgium) for measuring FBG sensors (1), a custommade guiding sheath with EMT sensors and integrated optical 


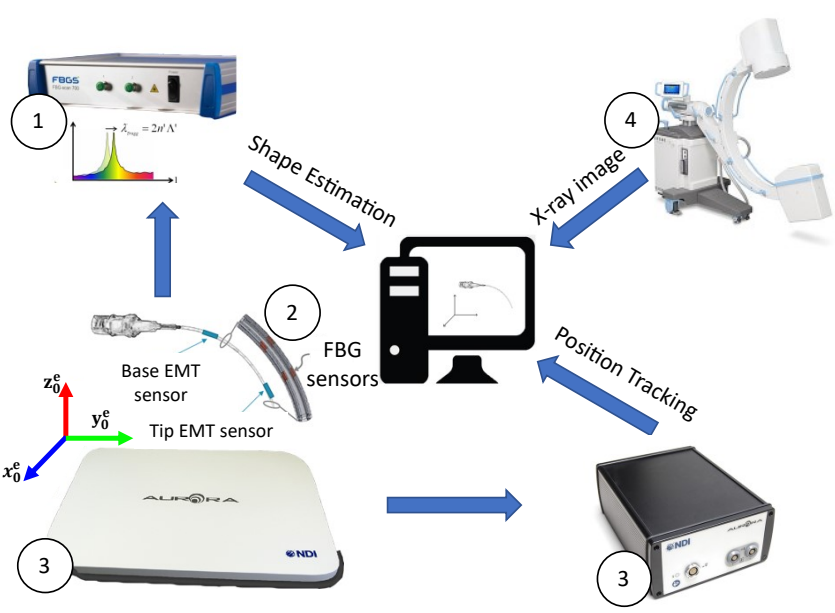

Fig. 1. Experimental platform which includes FBG interrogator for shape sensing and EMT system to localize the sensorized catheter in a fixed coordinate frame. X-ray image is incorporated to improve the shape sensing accuracy.

fiber that represents the interventional catheter (2), a table-top NDI Aurora EMT system (3) (Northern Digital Inc., Ontario, Canada) and fluoroscopic system (4). An 8.5 F Destino bidirectional steerable guiding sheath (Oscor, Düsseldorf, Germany) was prepared with two embedded 6-DOFs NDI Aurora EMT sensors. The first EMT sensor was approximately located at $5 \mathrm{~mm}$ from the catheter tip. The second EMT sensor was placed approximately at $170 \mathrm{~mm}$ from the tip EMT sensor. The physical length of an 6-DOFs EMT sensor is $0.8 \mathrm{~mm} \times$ $0.9 \mathrm{~mm}$, but the manufacturer does not provide the exact location on the sensor that is measured. The co-registration step described in sec. III-B, is established to find out exactly where the measurement locations are on the EMT sensors and the correspondence between the measured locations and the reconstructed shape. A $250 \mu \mathrm{m}$ four-core fiber consisting of 68 FBGs from FBGS (Geel, Belgium) is integrated the catheter. Each core contains 17 gratings distributed $10 \mathrm{~mm}$ apart from each other. Each grating has a different center wavelength. The length of each FBG sensor is $4 \mathrm{~mm}$. Two 3D printed (Vero White RGD835 by Stratasys) were used to hold the four-core fiber in the center. Figure 2 shows how the 2 EMT sensors are positioned relative to the catheter body.

\section{FUSION FRAMEWORK FOR DYNAMIC TWIST COMPENSATION}

The underlying idea of the proposed framework consists of online estimating the strain induced by the dynamic twist ( $\left.\varepsilon_{\text {Twist }}\right)$ and compensating for twist by subtracting the twistinduced strain from the measured wavelength shift. Figure 3 shows the overall flowchart of the proposed framework. The shape reconstruction algorithm starts by measuring the wavelength shift. The strains of the outer cores $\varepsilon_{i \in\{1,2,3\}}$ are updated by subtracting the central core strain and the twistinduced strain $\varepsilon_{\text {Twist }}$. The newly calculated strains are then interpolated and used to reconstruct the 3D shape of the catheter. After that, the 3D shape is localized in the EMT coordinate frame. This is done by incorporating the information from the

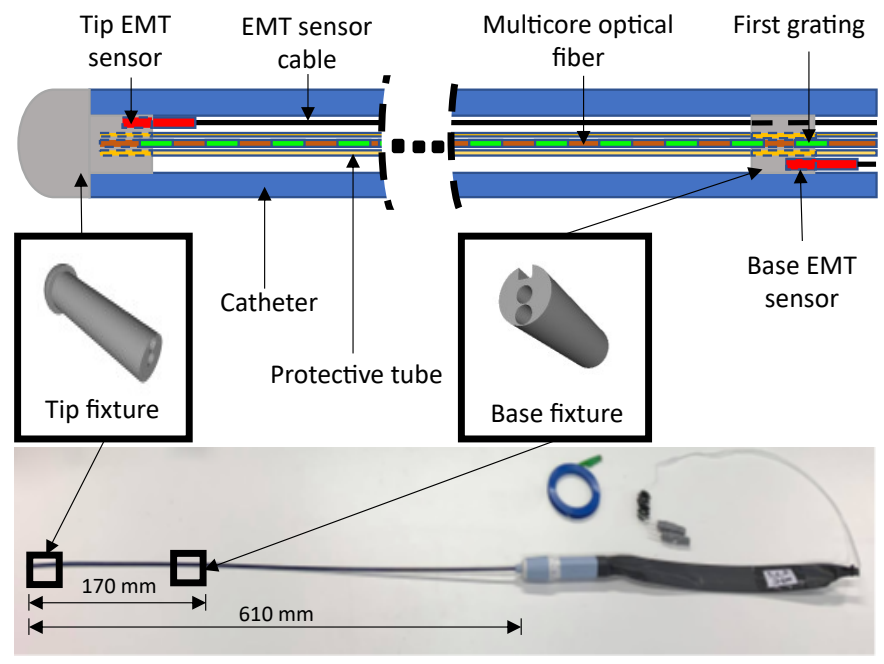

Fig. 2. Schematic of the proposed catheter design. The catheter is equipped with two EMT sensors. A multi-core optical fiber is fixed to the center channel by two 3D printed fixtures.

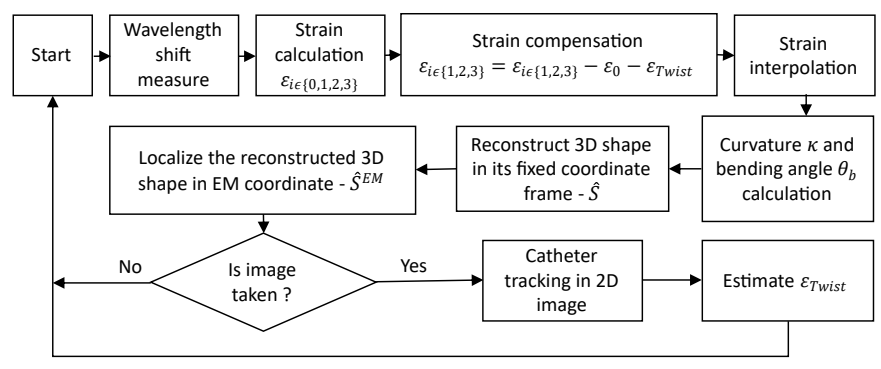

Fig. 3. Overall flowchart of the fusion framework. The twist-induced strain is figured out by fusing the FBG-based shape estimate with 2D images. The calculated strain will be used to compensate for the twist until new image is available.

2 EMT sensors. The FBG-based shape reconstruction method and the spatial calibration that is conducted to localize the catheter shape in the EMT coordinate system are described in sec. III-A and sec. III-B, respectively. In case there is a new fluoroscopic image available, the twist estimation process is carried out to update the estimate of the dynamic twist-induced strain. The fusion framework uses the shape reconstructed from the FBG and EMT as an initial estimate. A method originally introduced by Chang et al. [20] (summarized in sec. III-D) is then used to track the catheter shape in the 2D fluoroscopic image plane departing from the shape that was estimated in the previous step. Next, an optimization problem is solved. The dynamic twist $\varepsilon_{\text {Twist }}$ is found as the value that makes the reconstructed shape correspond maximally to the shape detected in the 2D fluoroscopic image. This optimization problem is detailed in sec. III-E. Based upon the updated twist, an improved 3D shape estimate is then computed and so on. It is important to note that the optimization also improves the shape sensing at moments when no new fluoroscopic images are produced.

\section{A. FBG-based shape reconstruction}

FBGs are distributed Bragg reflectors that reflect a narrow band of the spectrum of incoming light while the belonging to 
other ranges are lossless transmitted. When multiple gratings are used, typically each their own reflected wavelength. The gratings are fabricated in such a manner that each central wavelength is separated sufficiently far from the other. The reflected light from one grating can therefore be distinguished easily from the light that is reflected by the other gratings. The reflected spectrum of one grating changes due to temperature variation, mechanical strain or torsion (twist) applied on the optical fiber. An interrogator connected to the optical fiber measures the central wavelength of the reflected light. Based on the computed wavelength shift, the instantaneous curvature is then estimated. A curvature-strain model which relates the fiber strain and the curvature is described as

$$
\begin{gathered}
\varepsilon_{0}=\varepsilon_{\text {Temp }}+\varepsilon_{\text {Axial }}, \\
\varepsilon_{i \in\{1,2,3\}}=\varepsilon_{\text {Bend }_{i}}+\varepsilon_{\text {Twist }}+\varepsilon_{\text {Temp }}+\varepsilon_{\text {Axial }}, \\
\varepsilon_{\text {Bend }_{i}}=-\kappa r \cos \left(\theta_{b}-\frac{3 \pi}{2}-\theta_{i}\right) .
\end{gathered}
$$

where $\varepsilon_{0}$ is the strain in the central core and $\varepsilon_{1}, \varepsilon_{2}$ and $\varepsilon_{3}$ are the strain in the $1^{\text {st }}, 2^{\text {nd }}$ and $3^{\text {rd }}$ core in the case of a four-core fiber. The parameter $r$ is the distance from each outer core to the central core, $\theta_{i}$ is the angle of $i^{t h}$ core with respect to the $\mathrm{x}$ axis as shown in Fig. 4 (b) (e.g. $\theta_{i}$ could be $-30^{\circ}, 90^{\circ}$ and $210^{\circ}$ in the four-core fiber), $\kappa$ is the curvature and $\theta_{b}$ is the direction of the bending plane. The strain due to temperature $\varepsilon_{T e m p}$ and the axial strain $\varepsilon_{\text {Axial }}$ due to a longitudinal load aligned to the fiber longitudinal axis can be known by measuring the wavelength shift in the central core when it is assumed to with the neutral line of the fiber. The bend-induced strain of the $i^{t h}$ core is $\varepsilon_{\text {Bend }_{i}}$. The twist-induced strain $\varepsilon_{\text {Twist }}$ is incorporated into the curvature-strain model of (2) to take into account the effect of varying applied on the catheter during the procedure. Hypothesizing that the fiber has an ideal elastic behavior, plane sections remain plane at all times, radii remain straight and cross-sections remain plane and circular, SaintVenant's Torsion Theory can be applied for the optical fiber. The twist-induced strain varies linearly with $r$. In other words, the twist is proportional to the radial distance. So at the neutral line (and central core), the twist is zero, and at each core which is assumed to be located at the same distance from the neutral line, it is thus equal. Traditionally, $\varepsilon_{i \in\{1,2,3\}}-\left(\varepsilon_{T e m p}+\varepsilon_{\text {Axial }}\right)$ is used to compute $\kappa$ and $\theta_{b}$ in (3), but if $\varepsilon_{\text {Twist }}$ is not excluded this will give rise to an error on both $\kappa$ and $\theta_{b}$. Numerical method can be suggested to solve the set of equation (1)-(3). However, since this is an ill-conditioned problem, the twistinduced strain estimation process, described in sec. III-E is derived to address this problem.

The shape reconstruction based on FBGs relies on curvature and torsion estimated at the discrete grating locations. A typical FBG-based shape reconstruction process, as described in [7], consists of the following steps:

1) wavelength shift measurement;

2) strain computation over each core (in this case 18);

3) strain interpolation for all discrete points;

4) curvature and bending angle computation;

5) space curve shape reconstruction.
After these steps, a shape is thus obtained. At this point, this shape is relative to the first grating. To determine where this $1^{\text {st }}$ grating and the remaining shape lie in space, a co-registration with EMT must be conducted. This method is explained in the next section.

\section{B. EMT - FBG co-registration}

The pose of each EMT sensor in the EMT coordinate frame is defined as

$$
\mathbf{T}^{e}=\left[\begin{array}{cc}
\mathbf{R}_{3 \times 3}^{e} & \mathbf{p}_{3 \times 1}^{e} \\
0_{1 \times 3} & 1
\end{array}\right]
$$

where $\mathbf{R}^{e}=\left[\begin{array}{lll}\mathbf{x}^{e} & \mathbf{y}^{e} & \mathbf{z}^{e}\end{array}\right]$ is a $3 \times 3$ rotation matrix and $\mathbf{p}^{e}$ describes the current position of the EMT sensor. Both are expressed relative to a coordinate frame $\{e\}$ that is fixed to the EMT generator which serves as world reference frame in this work. The relation between the different coordinate frames is shown in Fig. 4(a).

The 3D shape of the catheter is reconstructed by the method of sec. III-A. The reconstructed shape in reference frame $\{f\}$ fixedly attached to the first FBG grating is expressed as a set of $n$ points $\mathbf{S}^{f}=\left[\begin{array}{llll}\mathbf{s}_{\mathbf{1}} & \mathbf{s}_{2} & \ldots & \mathbf{s}_{\mathbf{n}}\end{array}\right]$ where $\mathbf{s}_{\mathbf{i}}$ is the coordinate of each point together with a set of tangent vectors $\mathbf{V}^{f}=$ $\left[\begin{array}{llll}\mathbf{v}_{\mathbf{1}} & \mathbf{v}_{\mathbf{2}} & \ldots & \mathbf{v}_{\mathbf{n}}\end{array}\right]$ where $\mathbf{v}_{\mathbf{i}}$ is the tangent vector at the point $\mathbf{s}_{\mathbf{i}}$. A spatial calibration step needs to be carried out to find the correspondence between the measured shape $\mathbf{S}^{f}$ and the measured pose $\mathbf{T}^{e}$ of the base and tip EMT sensors.

Jäckle et al. used computer tomography (CT) scans and optical markers together with a point-based registration algorithm to align the reconstructed shape $\mathbf{S}^{f}$ to the segmented catheter shape in the CT scan [19]. Afterward, the corresponding arc length of tip $\left(l_{\text {Tip }}\right)$ and base $\left(l_{\text {Base }}\right)$ EMT sensor were manually obtained. In this paper, we propose a procedure that uses only the reconstructed shape from FBG together with the poses from 2 EMT sensors to conduct the co-registration. For this co-registration, the catheter with 2 EMT sensors and multi-core FBG fiber is fixed at the base at the level of the proximal EMT sensor and is subsequently bent on a plane in 2 configurations. Here, it is important that the configuration should be symmetric relative to the straight configuration as shown in Fig. 5(a). Since the FBG-based shape reconstruction method provides the 3D shape of the catheter, the distance between each corresponding point at a given arc length $l$ along the fiber can be measured. This distance can be set out for each point along the length of the catheter. Also, the Euclidean distance between the tip EMT sensor pose can be calculated in the 2 configurations. By comparing the traveled EMT distance with the interpolated distance traveled by the fiber, the corresponding arc length $l_{\text {Tip }}$ of the tip EMT sensor relative to the FBG grating can be determined. The distance between the tip and the base EMT sensor can be measured in a straight configuration, as distance between two EMT sensors positions. From the tip position of the catheter in the straight configuration, the length $l_{\text {Tip }}$ and the space between two EMT sensors, the arc length of the base EMT sensor relative to $\{f\}$, $l_{\text {Base }}$ can then be estimated.

Note that while we assume that the EMT sensors are aligned parallel to the catheter's centerline, the EMT sensors are 


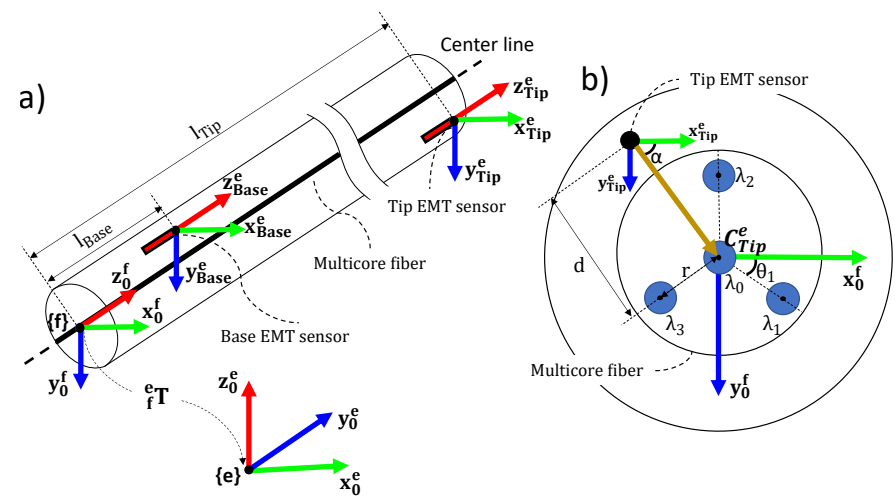

Fig. 4. a) Relationship between EMT coordinate frame $\{e\}$ and fixed coordinated frame $\{f\}$ that is attached to the first FBG grating; b) cross sectional view at the tip of the catheter view of a four-core fiber.

generally not collinear to that centerline. Therefore for each EMT sensor $j$ where $j$ can be the base or the tip EMT sensor, a calibration step is needed to find the transformation matrix $\mathbf{T}_{\text {Calib }}$ to transform the relative position of this EMT sensor to the centerline:

$$
\left[\begin{array}{c}
\mathbf{s}^{e} \\
1
\end{array}\right]=\mathbf{T}_{\text {Calib }}\left[\begin{array}{c}
\mathbf{p}^{e} \\
1
\end{array}\right]
$$

where

$$
\mathbf{R}_{\text {Calib }}=\mathbf{R}^{e} \operatorname{Rot}_{z}(\alpha)=\left[\begin{array}{lll}
\mathbf{x}_{\text {Calib }} & \mathbf{y}_{\text {Calib }} & \mathbf{z}_{\text {Calib }}
\end{array}\right],
$$

which gives $\mathbf{x}_{C a l i b}$ that is used to find the transformation

$$
\mathbf{T}_{\text {Calib }}=\left[\begin{array}{cc}
\mathbf{I}_{3 \times 3} & d \mathbf{x}_{\text {Calib }} \\
0 & 1
\end{array}\right] .
$$

The angle between the x-axis of the EMT sensor and the vector from $\mathbf{p}^{e}$ to $\mathbf{s}^{e}$ is defined as $\alpha$. Note that $d$ in (7) is the distance from the EMT sensor to the centerline. The relation between these parameters can be seen in Fig. 4(b). To estimate $T_{\text {Calib }}$ as explained next, the catheter in a straight configuration will be rotated about its axis. A straight 3D printed tube that has an inner diameter of $4.2 \mathrm{~mm}$ (same as the outer diameter of the catheter sheath) was made and fixed on top of the EMT field generator. In such case the catheter sheath was inserted into the 3D tube and rotated, making sure that only pure rotation was achieved. The position measurements $\mathbf{p}^{e}$ from each EMT sensor should follow a circular pattern with center $\mathbf{C}^{e}$. An optimization problem can then be formulated to find $\alpha$ and $d$ by minimizing the following cost function

$$
\underset{d_{j}, \alpha_{j}}{\operatorname{argmin}} \frac{1}{n} \sum_{i=1}^{n}\left(\mathbf{p}_{i_{j}}^{e}-\mathbf{s}_{i_{j}}^{e}\right) \quad j \in\{\text { Base,Tip }\},
$$

where $n$ is the number of position samples gathered during rotating the catheter around its axis for each EMT sensor.

To localize the position of the catheter's shape reconstructed by FBG in the EMT coordinate, a method to register two coordinate frames proposed by Horn is used [21]. Four shape points in the fixed shape sensing coordinate relative to the first FBG grating $\{f\}$ :

$$
\mathbf{N}^{f}=\left\{\mathbf{s}_{\text {Base }}^{f}, \mathbf{s}_{\text {Base }}^{f}+\mathbf{v}_{\text {Base }}^{f}, \mathbf{s}_{\text {Tip }}^{f}, \mathbf{s}_{\text {Tip }}^{f}+\mathbf{v}_{\text {Tip }}^{f}\right\},
$$
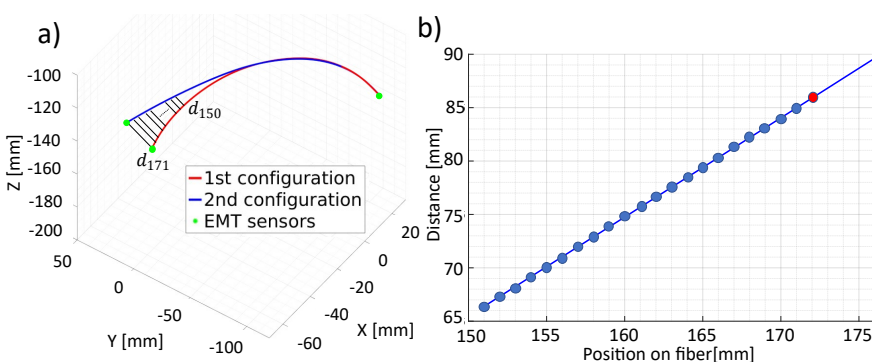

Fig. 5. a) 3D shape reconstruction of 2 configurations in fixed FBG coordinate, b) blue points show the distance between each corresponding point at a given arc length $l$ along the fiber in the $1^{\text {st }}$ configuration and $2^{\text {nd }}$ configuration, red point is the measured point of the tip EMT sensor.

together with their corresponding points in the EMT frame $\{e\}$ :

$$
\mathbf{N}^{e}=\left\{\mathbf{s}_{\text {Base }}^{e}, \mathbf{s}_{\text {Base }}^{e}+\mathbf{v}_{\text {Base }}^{e}, \mathbf{s}_{\text {Tip }}^{e}, \mathbf{s}_{\text {Tip }}^{e}+\mathbf{v}_{\text {Tip }}^{e}\right\}
$$

are used to localize the catheter shape $\mathbf{S}^{e}$ in the EMT frame. A rigid transformation matrix ${ }_{f}^{e} T$ to transform the catheter shape in the fixed shape sensing coordinate to the EMT coordinate calculated with the registration method between 2 frames proposed by [21].

$$
\mathbf{S}^{e}={ }_{f}^{e} \mathbf{T} \mathbf{S}^{f}
$$

\section{B-spline centerline model}

The catheter shape is represented by a B-spline curve to limit the shape space and decrease the high dimensionality as shown in [20]. This model-based catheter representation also makes the shape detection in the $2 \mathrm{D}$ image more robust and able to deal with noise and missing measurements (e.g. part of the catheter is occluded). The standard basis function representation of a $\mathrm{B}$-spline curve of degree $k-1$ is given by:

$$
\mathbf{b}(\mathbf{t})=\sum_{i=1}^{n} \mathbf{C}_{\mathbf{i}} B_{i, k}(t)
$$

where $\mathbf{C}_{i} \in \mathbb{R}^{N}$ are the $\mathrm{n}$ control points and $B_{i, k}(t)$ are basis functions which can be derived by the De Boor-Cox recursive formula [22]. This work uses three-dimensional $(N=3)$ cubic open B-splines with degree $3(k=4)$ which are $\mathbb{C}^{2}$ continuously differentiable functions. For a cubic B-spline curve with four control points $\left[\mathbf{C}_{i-1}, \mathbf{C}_{i}, \mathbf{C}_{i+1}, \mathbf{C}_{i+2}\right]$, the local cubic B-spline can be defined as:

$\mathbf{b}_{i}(t)=\frac{1}{6}\left[\begin{array}{llll}t^{3} & t^{2} & t^{1} & t^{0}\end{array}\right]\left[\begin{array}{cccc}-1 & 3 & -3 & 1 \\ 3 & -6 & 3 & 0 \\ -3 & 0 & 3 & 0 \\ 1 & 4 & 1 & 0\end{array}\right]\left[\begin{array}{c}\mathbf{C}_{i-1} \\ \mathbf{C}_{i} \\ \mathbf{C}_{i+1} \\ \mathbf{C}_{i+2}\end{array}\right]$

The sampling parameter $t \in[0,1]$ is uniformly distributed for point interpolation. For point interpolation, a clamped cubic Bspline has $\mathbf{C}_{j \in-2, \ldots, n+3}$ and at the boundary $\mathbf{C}_{1}=\mathbf{C}_{j<1}$ and $\mathbf{C}_{n}=\mathbf{C}_{j>n}$. Instead of fitting the B-spline curve to a number of control points, the knot-driven B-spline representation is used to have better local control [22] in order to perform optimisation for region-based pixel-wise posterior. Given a set 
of knot points $\mathbf{K}_{i}$, the control points for a clamped cubic Bspline can be found by:

$$
\left[\begin{array}{c}
\mathbf{C}_{1} \\
\vdots \\
\mathbf{C}_{n}
\end{array}\right]=\left[\begin{array}{ccccccc}
1 & & & & & \cdots & 0 \\
\frac{1}{6} & \frac{2}{3} & \frac{1}{6} & & & & \\
& \ddots & \ddots & \ddots & & & \vdots \\
& & \frac{1}{6} & \frac{2}{3} & \frac{1}{6} & & \\
\vdots & & & \ddots & \ddots & \ddots & \\
0 & & & & \frac{1}{6} & \frac{2}{3} & \frac{1}{6} \\
\\
0
\end{array}\right.
$$

with $n \geqslant 4$. The number of control points and knot points are equal for the inverse transform and due to the clamp, $\mathbf{K}_{1}=\mathbf{C}_{1}$ and $\mathbf{K}_{n}=\mathbf{C}_{n}$ hold at the boundary.

\section{Catheter tracking in 2D image}

Several methods have been reported to register EMT tracking system and fluoroscopy [23], [24]. From this registration process, it is possible to compute a projection matrix that can be used to project the 3D shape expressed in the EMT coordinate frame in the image plane $\Omega$ of the fluoroscope. For the sake of brevity, we assumed that one of the above methods has been applied and that the projection matrix is known. The B-spline tube model in the $2 \mathrm{D}$ image $\Omega$ can thus be defined by a set of knot points sampling from the estimated shape from FBG followed by projecting to the $2 \mathrm{D}$ image plane. Then, the corresponding control points can be calculated by (14) and interpolated via (13) to obtain a $\mathrm{B}$-spline representation of the shape projected in the image plane. Currently, the estimated shape of the catheter in the image plane is represented by B-spline center-line model. However, the shape of the catheter captured by the fluoroscope is normally seen as a contour. Thus, a B-spline tube model $\mathbf{c}_{i}(t)$ is defined from the centerline model to track the catheter shape in the $2 \mathrm{D}$ image plane

$$
\mathbf{c}_{i \in\{1, \ldots, n\}}(t)=\mathbf{b}_{i}(t) \pm R\left[\begin{array}{cc}
0 & 1 \\
-1 & 0
\end{array}\right] \hat{\mathbf{W}}_{i}(t),
$$

where $n$ is the number of knot points sampled from the estimated shape by the FBG, $\hat{\mathbf{W}}_{i}=\frac{\mathbf{W}_{i}}{\left\|\mathbf{W}_{i}\right\|}$ and $\mathbf{W}_{i}=\frac{\partial \mathbf{b}_{i}}{\partial t}$ is the tangent vector at the point $\mathbf{b}_{i}(t)$. The radius of the tube is set as radius. Since the tube has the same geometrical dimensions and is projected into the image plane with a known scale factor (this can be known by registration process of EMT and fluoroscopy). The radius can be chosen based on the radius of the catheter. An example of the proposed Bspline tube model in the 2D image plane is shown in Fig. 6. The contour $\mathbf{c}=\left\{\mathbf{c}_{1}, \ldots, \mathbf{c}_{n}\right\}$ delimits the foreground $\Omega_{f}$ and background $\Omega_{b}$ regions. These two regions are associated to the non-parametric foreground and background appearance models $P\left(\mathbf{y} \mid M_{f}\right)$ and $P\left(\mathbf{y} \mid M_{b}\right)$, respectively where $y$ represents the pixel value at pixel position $x$ in the image coordinate frame. $M=\left\{M_{f}, M_{b}\right\}$ is a model parameter that indicates either foreground or background.

The pixel-wise posteriors $P(\mathbf{c} \mid \Omega)$ is the probability of the catheter contour $c$ given all pixel data $\Omega$. According to [20], the joint probability for a single pixel given by the joint graphical model shown in Fig. 6(e) can be found as

$$
P(\mathbf{x}, \mathbf{y}, \mathbf{c}, M)=P(\mathbf{x} \mid \mathbf{c}, M) P(\mathbf{y} \mid M) P(M) P(\mathbf{c}) .
$$

Equation (16) is now divided by:

$$
P(\mathbf{y})=P\left(\mathbf{y} \mid M_{f}\right) P\left(M_{f}\right)+P\left(\mathbf{y} \mid M_{b}\right) P\left(M_{b}\right)
$$

to give:

$$
P(\mathbf{x}, \mathbf{c}, M \mid \mathbf{y})=P(\mathbf{x} \mid \mathbf{c}, M) P(M \mid \mathbf{y}) P(\mathbf{c}),
$$

where the term $P(M \mid \mathbf{y})$ is the pixel-wise posterior, of the model $M$, given a pixel value $\mathbf{y}$ :

$$
P\left(M_{j=\{f, b\}} \mid \mathbf{y}\right)=\frac{P\left(\mathbf{y} \mid M_{j}\right) P\left(M_{j}\right)}{P(\mathbf{y})} .
$$

Marginalising (18) over the model $M$, the pixel-wise posterior probability of contour $\mathbf{c}$ given a pixel $\mathbf{x}$ with value $\mathbf{y}$ is defined as:

$$
P(\mathbf{c} \mid \mathbf{x}, \mathbf{y})=\frac{1}{P(\mathbf{x})} \sum_{i=f, b}\left\{P\left(\mathbf{x} \mid \mathbf{c}, M_{i}\right) P\left(M_{i} \mid \mathbf{y}\right)\right\} P(\mathbf{c}) .
$$

The overall pixel-wise posterior of the catheter contour $\mathbf{c}$ given all pixel data $\Omega$ is expressed as

$$
P(\mathbf{c} \mid \Omega)=\prod_{j=1}^{N}\left[\sum_{i=f, b}\left\{P\left(\mathbf{x} \mid \mathbf{c}, M_{i}\right) P\left(M_{i} \mid \mathbf{y}\right)\right\}\right] P(\mathbf{c})
$$

This pixel-wise posterior takes into account every single pixel in the foreground and the background. Taking advantage of modeling the likelihood of foreground and background by a non-parametric probability distribution using a histogram, this probabilistic framework can be utilized with several types of prior knowledge. In the proposed approach, the values and Frangi filtering magnitudes of the image are used as the measurements. Frangi filter helps to enhance vessels in images by examining the multiscale second order local structure of an image proposed by Frangi et al. [25].

The initial estimated tube model in the $2 \mathrm{D}$ image plane is defined by the contour and the probabilistic framework (21) allow tracking to be performed by maximizing the posterior of contour $\mathbf{c}$ with respect to the knot points $\mathbf{K}_{i}$. The log posterior of $(21)$ is

$$
\stackrel{\circ}{\mathbf{c}}=\underset{\mathbf{c}}{\operatorname{argmax}} \sum_{i=1}^{N} \log \left\{P\left(\mathbf{x}_{i} \mid \mathbf{c}, \mathbf{y}_{i}\right)\right\}+\log (P(\mathbf{c})),
$$

where $P\left(\mathbf{x}_{i} \mid \mathbf{c}, \mathbf{y}_{i}\right)$ is defined as [4]

$\frac{H_{\varepsilon}\left(\Phi\left(\mathbf{x}_{i}\right)\right) P\left(\mathbf{y}_{i} \mid M_{f}\right)+\left(1-H_{\varepsilon}\left(\Phi\left(\mathbf{x}_{i}\right)\right)\right) P\left(\mathbf{y}_{i} \mid M_{b}\right)}{\sum_{i=1} H_{\varepsilon}\left(\Phi\left(\mathbf{x}_{i}\right)\right) P\left(\mathbf{y}_{i} \mid M_{f}\right)+\sum_{i=1}^{N}\left(1-H_{\varepsilon}\left(\Phi\left(\mathbf{x}_{i}\right)\right)\right) P\left(\mathbf{y}_{i} \mid M_{b}\right)}$.

The $\Phi(\mathbf{x})$ is a signed distance function such that

$$
\Phi(\mathbf{x})= \begin{cases}0 & \text { if } \mathbf{x} \in \mathbf{c} \\ \text { dist } & \text { if } \mathbf{x} \in \Omega_{f} \\ - \text { dist } & \text { if } \mathbf{x} \in \Omega_{b}\end{cases}
$$



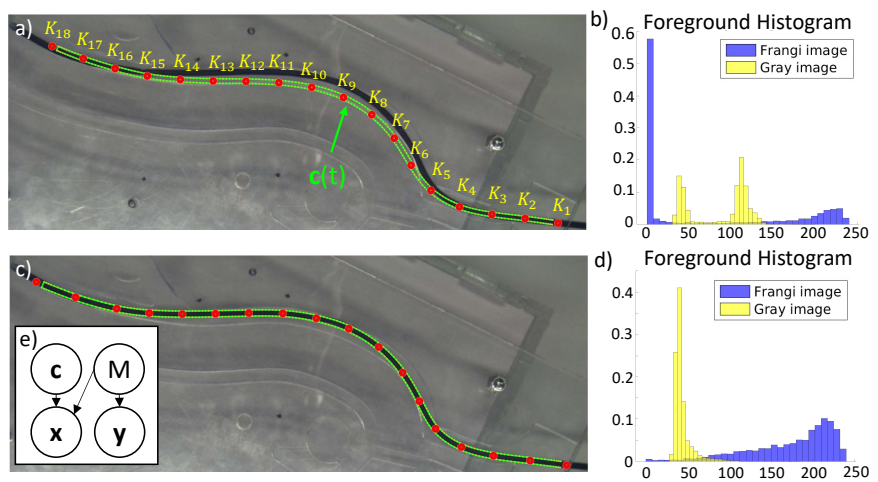

Fig. 6.

where dist is the Euclidean distance between a pixel at location $\mathbf{x}$ and the nearest pixel belonging to the contour $\mathbf{c}$. The function $H_{\varepsilon}$ is a smooth Heaviside function:

$H_{\varepsilon}(\Phi(\mathbf{x}))= \begin{cases}\frac{1}{2}\left(1.0+\frac{\Phi(\mathbf{x})}{\varepsilon}+\frac{1}{\pi} \sin \left(\frac{\Phi(\mathbf{x}) \pi}{\varepsilon}\right)\right) & \text { if }-\varepsilon \leq \Phi(\mathbf{x}) \leq \varepsilon \\ 0 & \text { if } \Phi(\mathbf{x})<-\varepsilon \\ 1 & \text { if } \Phi(\mathbf{x})>\varepsilon\end{cases}$

To optimize (22), we use a Gauss-Newton algorithm as suggested by [20]. The update $\Delta \mathrm{x}$ for each knot point is obtained as:

$$
\Delta \mathbf{x}=\left[\sum_{m=1}^{|\Omega|} B_{m}^{2} \mathbf{J}_{m}^{T} \mathbf{J}_{m}\right] \sum_{m=1}^{|\Omega|} \mathbf{J}_{m}^{T} B_{m},
$$

where $|\Omega|$ is the number of pixel and the Jacobian at each pixel being expressed as:

$$
\mathbf{J}_{m}=\left.\frac{\delta H_{\epsilon}}{\delta \Phi} \frac{\delta \Phi}{\delta \mathbf{x}}\right|_{\mathbf{x}=\mathbf{x}_{m}}=\delta_{\varepsilon}(\Phi) \nabla \Phi\left(\mathbf{x}_{m}\right)
$$

and

$$
B_{m}=\frac{P\left(\mathbf{y}_{i} \mid M_{f}\right)-P\left(\mathbf{y}_{i} \mid M_{b}\right)}{P\left(\mathbf{y}_{i} \mid M_{f}\right) H_{\varepsilon}\left(\mathbf{x}_{m}\right)+P\left(\mathbf{y}_{i} \mid M_{b}\right)\left(1-H_{\varepsilon}\left(\mathbf{x}_{m}\right)\right)} .
$$

Utilizing the $\delta_{\varepsilon}$ function makes only pixels that lie inside a narrow range affect the Jacobian. So, an update for each knot point uses the sum of $\Delta \mathbf{x}$ calculated from point $\mathbf{x}$ around the point belong to the contour. Figure 6 shows an example of the proposed catheter tracking in the $2 \mathrm{D}$ image plane. .

\section{E. Dynamic twist-induced strain estimation}

With all the above preliminary preparation, the dynamic twist can now be estimated by fusing the initial shape reconstructed by the FBG shape sensing and its correct projection in the 2D image plane obtained from sec. III-D. An optimization problem is formulated to vary the dynamic twist $\varepsilon_{\text {Twist }}$ so that the $2 \mathrm{D}$ projected shape from the knot points matches the shape that resulted from the catheter tracking in the image plane. The final bend-induced strains are calculated as

$$
\varepsilon_{\text {Bend }, i \in\{1,2,3\}}=\varepsilon_{i}-\varepsilon_{0}-\varepsilon_{\text {Twist }} .
$$

The twist-induced strain vector $\varepsilon_{\text {Twist }}$ is estimated by minimizing the cost function

$$
\arg \min _{\varepsilon_{\text {Twist }}} D\left(\varepsilon_{\text {Twist }}\right)+a E_{\text {Localize }}\left(\varepsilon_{\text {Twist }}\right),
$$

where the term $D$ is the distance between the catheter shape in the image plane calculated in the catheter tracking in 2D image step and the projection of the 3D shape reconstructed from the newly calculated strains from (27). $E_{\text {Localize }}$ is the localization error of the computed catheter shape in the EMT coordinate frame. The scaling factor $a$ regulates the relative effect between these terms. $D$ is defined by

$$
D=\frac{1}{n} \sum_{i=1}^{n} G^{2}\left(\operatorname{proj}\left(\mathbf{s}_{i}^{e}\right)\right)
$$

where $n$ is the total number of points used to represent the catheter centerline model in EMT coordinates. The term $\operatorname{proj}\left(\mathbf{s}_{i}^{e}\right)$ is the projection of the catheter shape in the 2D image plane. $G$ is the function that expresses the distance between $\operatorname{proj}\left(\mathbf{s}_{i}^{e}\right)$ and its closest point in the 2D image of catheter tube model found from the fluoroscopy-based catheter tracking. This distance is calculated by the Euclidean distance. The term $E_{\text {Localize }}$ is calculated as a sum of squares of residual errors:

$$
E_{\text {Localize }}=\frac{1}{4} \sum_{i=1}^{4}\left\|\mathbf{N}_{i}^{e}-_{f}^{e} \mathbf{T N}_{i}^{f}\right\|^{2}
$$

Using this approach, the twist-induced strain $\varepsilon_{\text {Twist }}$ is estimated such that it maximizes the correspondence between the reconstructed shape $\mathbf{S}^{e}$ with the catheter shape in the 2D image plane and the poses of EMT sensors in EMT coordinate frame. Note that the newly estimated $\varepsilon_{\text {Twist }}$ will be used advantageously throughout the upcoming time steps until the next fluoroscopic image is available.

The iterative approach to estimate $\varepsilon_{\text {Twist }}$ is not guaranteed to converge to the global optimum. However, experiments reported in sec. IV support that incorporating fluoroscopic images and EMT pose measurements effectively improves the accuracy of the overall shape reconstruction.

\section{EXPERIMENTAL ENVIRONMENT}

\section{A. Experimental setup for $2 D$ and $3 D$ verification}

To evaluate the proposed approach, three experiments were carried out in a lab setup. The first experiment was designed to verify the efficiency of the method in an in-plane bending case. The catheter was placed on top of a table-top NDI Aurora EMT field generator. A monocular Prosilica camera (Allied Vision Technology, Germany) was positioned approximately $50 \mathrm{~cm}$ above the setup, facing downward to mimic the fluoroscopy. The camera image plane was parallel to the plane of the EMT field generator. The catheter detailed in sec. II was used for this experiment. During the experiment, the catheter bent in 2 directions in a plane that parallel to the camera's image plane as shown in Fig. 10(a). This allows maximizing the area captured by the camera. Therefore, the image ensured to incorporate as much spatial information as possible. The camera images recorded at 30 frames per second (fps) while the catheter bending.

The second and the third experiment were carried out to evaluate the effect of the algorithm in 3D and a retraction was conducted for this purpose. In this experiment, two tubes with predefined shapes, given as mathematical functions were $3 \mathrm{D}$ 


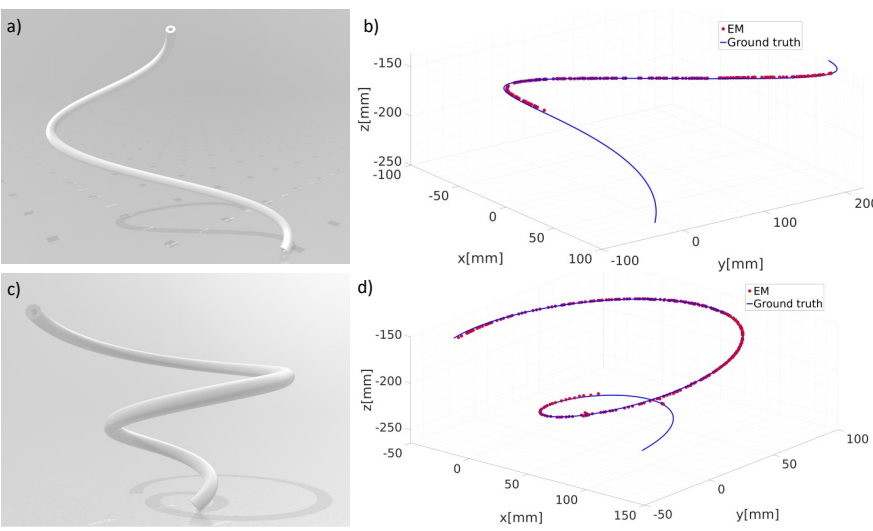

Fig. 7. a) c) The designs of the 3D printed tubes for 3D validation; b) d) the 3D printed tubes were registered to the EMT coordinate frame by sliding an EMT sensor along the tubes. The red points are EMT positions gathered during this action.
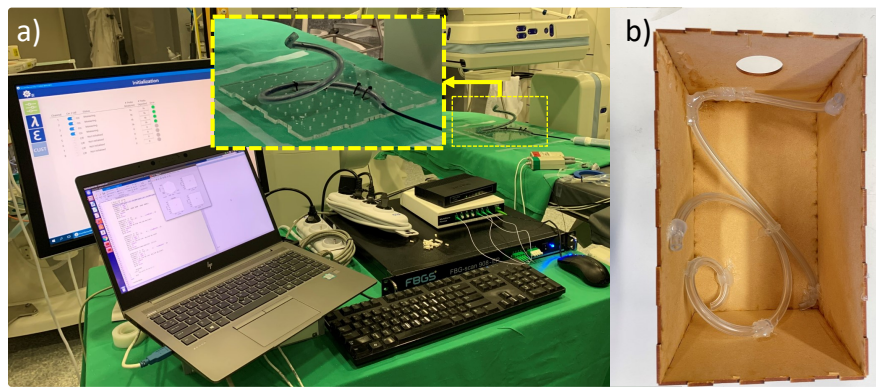

Fig. 8.

printed. The 3D printed tubes were made of TuskXC2700T with a resolution of $0.20 \mathrm{~mm}$ by Materialise (Leuven, Belgium). A polishing process was used to increase the inner surface's smoothness of the 3D printed tubes to assure the catheter was completely coaxial with the tubes while moving inside. To mitigate the flexing of 3D printed tubes, they were placed in a box and silicone adhesive was used to constrain at various points along the tube length as shown in Fig. 8(b). The box was then fixed on top of the field generator to ensure that the tubes could not move during retraction. The designs of 3D printed tubes is shown in Fig. 7(a)(c). The inner diameter of the tubes $4.2 \mathrm{~mm}$ which closely fitted the outer diameter of the catheter sheath, The arc lengths of the tubes were $500 \mathrm{~mm}$ long. The catheter was retracted in the printed tubes instead of inserting in order to minimize the friction applied on the catheter. During the experiment, the wavelengths of the fiber and the pose measurements from the EMT sensors were recorded to reconstruct the shape of the catheter. .

\section{B. Registration of EMT coordinate and image frame}

A known size chessboard pattern was used to register the EMT coordinate and the image frame as shown in Fig. 9. First, the positions of four outer corners of the planar pattern were recorded in the EMT coordinate frame. The 3D coordinates of the inner corners of the pattern were calculated based on the real geometry of the pattern. This 3D set of coordinates is denoted as $\mathbf{O}_{3 \times n}^{e}=\left[\mathbf{m}_{1} \ldots \mathbf{m}_{n}\right]$. An image was obtained by the camera and undistorted by the camera distortion parameters

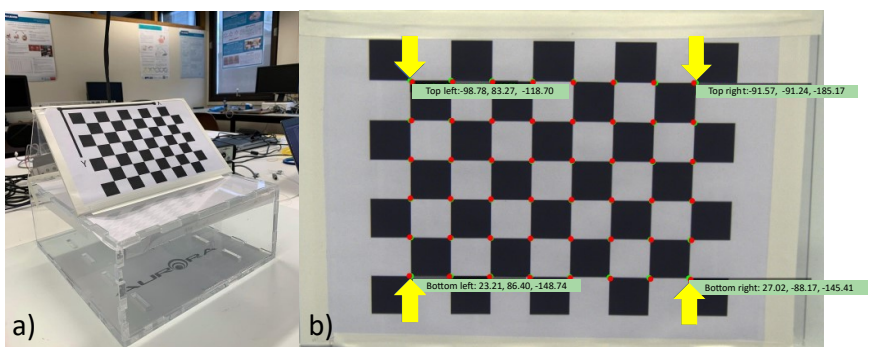

Fig. 9. a) Experimental setup for EMT - image plane registration; b) The red points are the projected points in the image frame, the yellow arrows show the measurement points of the EMT sensors.
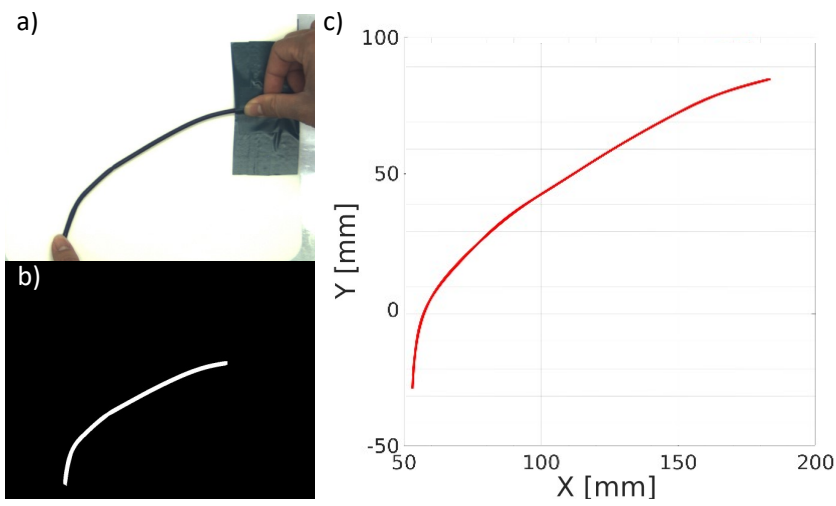

Fig. 10. a) Image captured by camera; b) result of color segmentation and skeletonization process; c) 3D ground truth shape in EMT coordinate frame.

[26]. A chessboard pattern detection algorithm [27] was performed to find coordinates of corner points in the undistorted image. The set of $2 \mathrm{D}$ corner coordinates in the image plane $\{i m\}$ is denoted as $\mathbf{Q}_{2 \times n}^{i m}=\left[\mathbf{q}_{1} \ldots \mathbf{q}_{n}\right]$. The matrix $\mathbf{Q}^{i m}$ is rearranged to make each column $i^{\text {th }}$ in $\mathbf{Q}^{i m}$ as a 2D projection of the $i^{t h}$ point in $\mathbf{O}^{e}$. The $2 \times 4$ projection matrix $\mathbf{P}$ to project the $3 \mathrm{D}$ coordinate from the EMT frame to the $2 \mathrm{D}$ image plane is obtained by solving the following optimization problem:

$$
\min _{\mathbf{P}}\left\|\mathbf{P}\left[\begin{array}{c}
\mathbf{O}_{3 \times n}^{e} \\
1_{1 \times n}^{e}
\end{array}\right]-\mathbf{Q}_{2 \times n}^{i m}\right\|,
$$

A unique optimal solution can be calculated with an appropriate number of points (at least 4 points should be used). In our experiments. 45 points were used to register the EMT coordinate frame with the image plane as shown in Fig. 9. The mean residual error was $0.59 \pm 0.05 \mathrm{~mm}$ and the maximum residual error was $1.56 \mathrm{~mm}$.

\section{Ground truth generation}

The ground truth data for evaluating the accuracy of our method in the first experiment generated from the images acquired with the camera. The images first processed to extract the catheter shape in 2D by color segmentation. Each contour in the image evaluated to find the contour that the largest area. This contour considered as the catheter's contour. The algorithm from Lee et al. used to skeletonize the contour [28]. The set of points that belonged to the skeleton was the 2D projected shape of the catheter in the image plane. An affine transformation performed to map this set of points to 
the EMT coordinate frame. Since the catheter bent in the $\mathrm{x}-\mathrm{y}$ plane, the $\mathrm{z}$ value should be constant. It determined by averaging the $\mathrm{z}$ value from the 2 attached EMT sensors. The $3 \mathrm{D}$ set of skeleton points then approximated with the B-spline curve presented in Section III-C. This spline treated as the ground truth. The ground truth generation process is described in Fig. 10.

In the second and third experiments, the 3D printed tubes were fixed on top of the field generator. Since the 3D printed tubes were made with predefined mathematical functions:

$$
\begin{aligned}
& x=49.525(2 \cos (t)-\cos (2 t)) \sin (2 t) \\
& y=53.025(2 \sin (t)-\sin (2 t)) \cos (2 t), \\
& z=100 t
\end{aligned}
$$

where $t=\left[\begin{array}{ll}0.775 \pi & 1.505 \pi\end{array}\right]$ in the second experiment and

$$
\begin{aligned}
& x=8 t \cos (t) \\
& y=8 t \sin (t), \\
& z=12 t
\end{aligned}
$$

where $t=\left[\begin{array}{ll}0.5 \pi & 3.8 \pi\end{array}\right]$ in the third experiment, the ground truth shapes of the catheter during the retraction process were known. The positions of the 3D tube in the EMT coordinate frame were determined by sliding an EMT sensor along the 3D tubes to gather position data as shown in Fig. 7(b)(d). This set of data allows the tube's positions in the EMT coordinate by using the above point-based registration method. The ground truth shapes provided by the mathematical functions were also used to generate idealistic fluoroscopic views of the scene. This was necessary because the $3 \mathrm{D}$ printed tubes were not transparent, which means that the catheter shapes during the retraction process could not be captured by the camera. The use of the virtual camera to simulate the fluoroscopic system also helps to exclude errors caused by the registration process between the 2 coordinate frames. The virtual camera was placed on top of the 3D printed tubes and the generated image size was set to $512 \times 512$ px.

\section{Evaluation methodology}

The reconstructed shape by our method is compared to the ground truth shape via the following metrics:

- the maximum distance error between the reconstructed and ground truth shape,

- the rms error, and

- the rms error along the respective $\mathrm{x}, \mathrm{y}$ and $\mathrm{z}$ axes of the EMT coordinate frame.

The distance error $d_{m i n}\left(\mathbf{S}_{g t}^{e}, \mathbf{S}_{e s t}^{e}(i)\right)$ is the distance between the $i^{t h}$ point of the estimated shape $\mathbf{S}_{e s t}^{e}$ to the closest point on the $\mathbf{S}_{g t}^{e}$ shape. In our error evaluation, two methods were compared. The error in shape reconstruction using only FBG (without twist compensation) and the shape sensing using the proposed method (i.e. shape reconstructed with the twist compensated strain) were compared.

\section{E. Results and discussion}

The spatial calibration step was first carried out to find the corresponding shape points $1_{\text {Base }}$ and $1_{\text {Tip }}$ of the 2 EMT sensors.
The calibration transformation matrix $\mathbf{T}_{\text {Calib }}$ determined for each EMT sensor to shift the EMT measurements to the corresponding points on centerline of the catheter. The results of the shape reconstruction in the in-plane bending experiment are shown in Fig. 11. Two approaches to decide when a fluoroscopic image should be taken were conducted. The first approach compares the error in the registration between the EMT and the shape reconstruction frame in the process of localization the reconstructed shape in the EMT frame $\left(E_{\text {Localize }}\right)$ with a predefined threshold value. If the registration error exceeds the threshold value, a fluoroscopic image would be commanded to re-estimate the twist-induced strain. In the in-plane bending experiment, the threshold was examined with two values $-0.7 \mathrm{~mm}$ and $0.9 \mathrm{~mm}$. . The lower the threshold value, the higher the number of fluoroscopic will be taken which leads to higher accuracy but also higher exposure to radiation. The second approach takes fluoroscopic images at a fixed rate. In the $3 \mathrm{D}$ verification experiments, the simulated image was generated at respectively $0.6 \mathrm{~Hz}$ and 0.3 $\mathrm{Hz}$. The time steps where fluoroscopic images were taken are marked with green dots in Fig. 11(b)(c). The proposed catheter shape reconstruction method able to run at $30 \mathrm{~Hz}$. By incorporating fluoroscopic measurements, the reconstructed shape improved. The catheter was first put in a straight configuration followed by bending left and right on the plane that parallel to the field generator. The green line shows the error obtained with improved FBG-based shape reconstruction method suggested by Al-Ahmad et al. [7], while the blue line and the magenta line show the errors obtained by the proposed framework with the threshold value of $0.7 \mathrm{~mm}$ and $0.9 \mathrm{~mm}$, respectively. The error in the improved FBG-based shape reconstruction algorithm increased when the catheter was bent left. The calibration for twist compensation suggested by Al-Ahmad et al. able to estimate the internal twist. This internal twist is defined as the amount of twist that presents even if the fiber is in an externally unloaded state, or is bent in a plane. To estimate the internal twist, the catheter was bent in one direction such that the entire shape sensing length is in-plane and under a certain curvature. By this calibration step, the bending angle due to intrinsic twist could be computed. This bending angle due to intrinsic twist is then used to compensate for the contribution of the intrinsic twist onto the bend angle $\theta_{b}$. The calibration for the internal twist compensation step suggested in [7] was performed on the experiment catheter by bending the catheter to the right-hand side on the plane that is parallel to the field generator. As it can be seen in Fig. 11(a)(b), the calibration for the internal twist compensation can help solve the twist problem for the bending direction in which the calibration was taken (i.e. when the catheter is bent right) but it does not address the problem completely as bending plane and orientation may change often and bending is not restricted to take place in one plane. By applying the proposed framework to estimate the twist-induced strain with the predefined threshold values of $0.7 \mathrm{~mm}$, both the median of the max distance error and the rms error decreased from $1.14 \mathrm{~mm}$ to $0.86 \mathrm{~mm}$ and from $0.52 \mathrm{~mm}$ to $0.39 \mathrm{~mm}$, respectively. Moreover, the errors were more consistent. The interquartile of the max distance error and the rms error of 


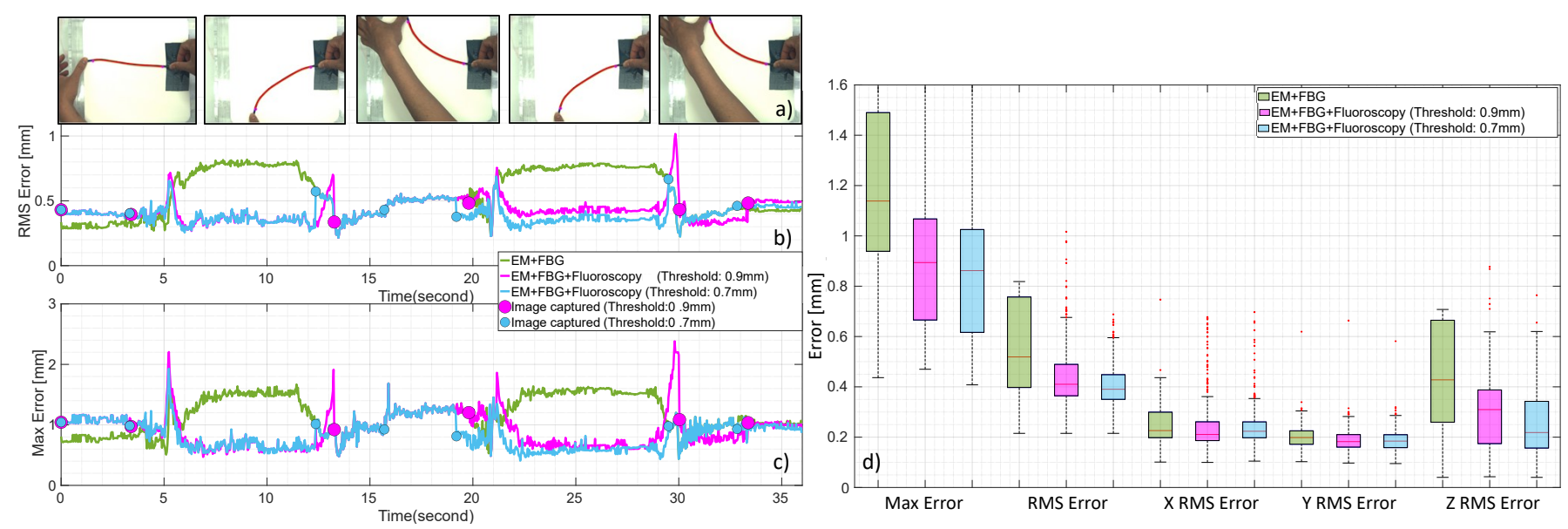

Fig. 11. In-plane bending experiment results a) images captured by camera; b) root mean square (rms) error and c) max distance error; $d$ ) analysis of each evaluation metric comparing reconstruction errors when using only EMT and FBG data and the here proposed framework (EMT + FBG + Fluoroscopy) of shape reconstruction for the in-plane experiment.
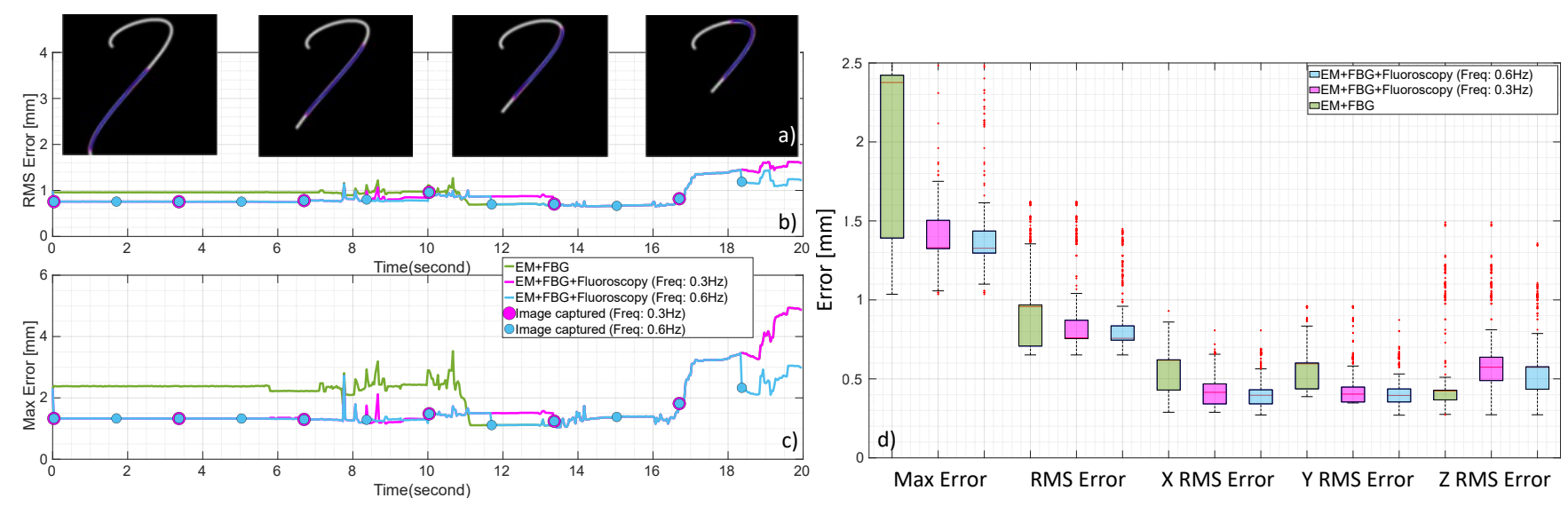

Fig. 12. First 3D experiment results a) simulated images; b) rms error and c) max distance error; d) analysis of each evaluation metric comparing the reconstruction errors when using only EMT and FBG data and the here proposed framework (EMT + FBG + Fluoroscopy) of shape reconstruction for the second 3D experiment.
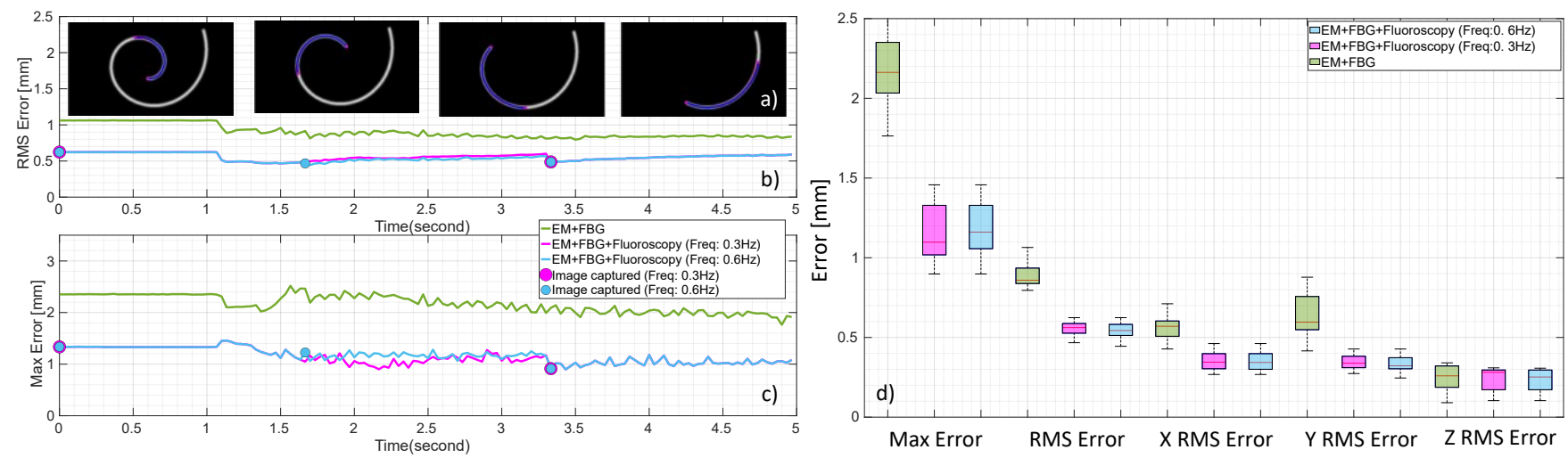

Fig. 13. Second 3D experiment results a) simulated images; b) rms error and c) max distance error; d) analysis of each evaluation metric comparing the reconstruction errors when using only EMT and FBG data and the here proposed framework (EMT + FBG + Fluoroscopy) of shape reconstruction for the third 3D experiment. 
the proposed framework were $0.41 \mathrm{~mm}$ and $0.10 \mathrm{~mm}$, while those of the shape reconstruction method in [7] were $0.55 \mathrm{~mm}$ and $0.36 \mathrm{~mm}$. The overall error in shape reconstruction also decreased in the case that the threshold value was set to $0.9 \mathrm{~mm}$. However, improved accuracy was achieved with the threshold value of $0.7 \mathrm{~mm}$ since a higher number of fluoroscopic images was taken. Note that respectively 7 and 6 images were captured when the threshold was set to $0.7 \mathrm{~mm}$ and $0.9 \mathrm{~mm}$ during 36 seconds bending in-plane experiment, respectively.

Figure 12 and 13 show the results of the two 3D bending experiments. The catheter was first inserted into the 3D printed tubes. The data was recorded during retraction. By incorporating the $2 \mathrm{D}$ images in the shape reconstruction, both the median of the max distance error and the median of the rms error in the second experiment decreased from $2.38 \mathrm{~mm}$ to $1.32 \mathrm{~mm}$ and from $0.96 \mathrm{~mm}$ to $0.76 \mathrm{~mm}$ with the image frequency setting at $0.6 \mathrm{~Hz}$, respectively. The same pattern can be seen in the third experiment. The median of the max distance error and median of rms error decreased from $2.16 \mathrm{~mm}$ to $1.16 \mathrm{~mm}$ and $0.86 \mathrm{~mm}$ to $0.54 \mathrm{~mm}$ with the image frequency setting at $0.6 \mathrm{~Hz}$. There was a slight increase in rms error along the $\mathrm{z}$-axis from $0.42 \mathrm{~mm}$ to $0.55 \mathrm{~mm}$ in the second experiment. It is believed that this is caused by the fact that the simulated camera was placed parallel to the natural plane of the 3D printed tube and the 2D image cannot capture much information of the catheter in the z-direction. In practice, this problem can be solved by choosing the orientation of the fluoroscope so that it maximizes the spatial information that is captured. The optimal orientation of the fluoroscope could be determined based on pre-operative models of the patient's anatomy. Moreover, biplane fluoroscopy could be used to capture images from different angles which in theory could further improve the performance of the fusion framework.

To figure out whether the incorporation of fluoroscopic images improves the shape sensing accuracy, statistical hypothesis testing was conducted. A Wilcoxon signed-rank test is used to compare the results of the proposed fusion framework and the traditional FBG-based shape sensing for max error and RMS error ( significant level is set to 0.05). For both in-plane bending and 3D experiments, the low p-values $\left(p_{w}\right)$ for max error and RMS error $\left(p_{w}<<0.05\right)$ show that incorporating fluoroscopic information significantly changes the shape sensing accuracy. The rms percentage error of our method $(0.32 \%)$ are comparable with the mean percentage error of Jäckle et al. [5] (0.3\%). similar pattern can be seen in max percentage error. However, the results in [5] obtained with static experiments. During the dynamic experiments, the catheter continuously underwent different strong bending configurations (with curvature up to $40 \mathrm{~m}^{-1}$ ). Thus, the results of our dynamic 3D experiments are more promising. Currently, the algorithm implemented in MATLAB (The MathWorks, Inc., Massachusetts, United States) and can run at $12 \mathrm{~Hz}$. The most time-consuming task is attributed to catheter tracking in 2D image step. In the future, Processing Unit (GPU) computing will be integrated to reduce the image processing time in order to increase the frequency of the proposed fusion framework.

\section{CONCLUSION}

In this work, a novel robust framework to reconstruct the 3D shape of a catheter during the procedure was introduced. A catheter consisting of a multi-core fiber and 2 EMT sensors was built and necessary spatial calibration steps conducted to localize the position of the reconstructed shape in the EMT coordinate were described. A method to estimate the twist-induced strain to improve the accuracy of the shape reconstruction by incorporating the sparse $2 \mathrm{D} \mathrm{X}$-ray images was proposed. The proposed framework was evaluated with 2D and 3D dynamic experiments.

With a sensorised length of $170 \mathrm{~mm}$, a percentage $\mathrm{rms}$ error of $0.23 \%$ in the $2 \mathrm{D}$ experiment and $0.32 \%$ in the 3D experiment were achieved. The performance exceeds the performance reported in all other works the authors are aware of. The sensed shape of the catheter can be overlaid on X-ray images. In this way, a clinician could navigate the catheter during a procedure without the need of continuous fluoroscopy or high-frame-rate fluoroscopy. Conventional 7.5FPS fluoroscopy is usually used during catheter ablation of atrial fibrillation. The report from Lee $e t$ al. showed that approximately $95 \%$ reduction was observed in radiation exposure quantified as dose area product by using 2-FPS instead of 7.5-FPS fluoroscopy [29]. Our proposed method allows the use of low-frame-rate fluoroscopy (approximately 1-FPS fluoroscopy) while providing continuous high accuracy position and shape information of the catheter for the interventionalists, which helps to reduce harmful exposure during procedure.

In future, animal experiments will be conducted to assess the performance of the proposed shape sensing algorithm invivo.

\section{REFERENCES}

[1] M. Wagner, S. Schafer, C. Strother, and C. Mistretta, " $4 d$ interventional device reconstruction from biplane fluoroscopy," Medical physics, vol. 43, no. 3, pp. 1324-1334, 2016.

[2] V. Sadick, W. Reed, L. Collins, N. Sadick, R. Heard, and J. Robinson, "Impact of biplane versus single-plane imaging on radiation dose, contrast load and procedural time in coronary angioplasty," The British journal of radiology, vol. 83, no. 989, pp. 379-394, 2010.

[3] S. Song, Z. Li, H. Yu, and H. Ren, "Electromagnetic positioning for tip tracking and shape sensing of flexible robots," IEEE Sensors Journal, vol. 15, no. 8, pp. 4565-4575, 2015.

[4] P. T. Tran, P.-L. Chang, H. De Praetere, J. Maes, D. Reynaerts, J. Vander Sloten, D. Stoyanov, and E. Vander Poorten, "3d catheter shape reconstruction using electromagnetic and image sensors," Journal of Medical Robotics Research, vol. 2, no. 03, p. 1740009, 2017.

[5] S. Jäckle, T. Eixmann, H. Schulz-Hildebrandt, G. Hüttmann, and T. Pätz, "Fiber optical shape sensing of flexible instruments for endovascular navigation," International journal of computer assisted radiology and surgery, vol. 14, no. 12, pp. 2137-2145, 2019.

[6] F. Khan, A. Denasi, D. Barrera, J. Madrigal, S. Sales, and S. Misra, "Multi-core optical fibers with bragg gratings as shape sensor for flexible medical instruments," IEEE sensors journal, vol. 19, no. 14, pp. 58785884, 2019.

[7] O. Al-Ahmad, M. Ourak, J. Van Roosbroeck, J. Vlekken, and E. Vander Poorten, "Improved fbg-based shape sensing methods for vascular catheterization treatment," IEEE Robotics and Automation Letters, vol. 5, no. 3, pp. 4687-4694, 2020.

[8] S. Sefati, M. Pozin, F. Alambeigi, I. Iordachita, R. H. Taylor, and M. Armand, "A highly sensitive fiber bragg grating shape sensor for continuum manipulators with large deflections," in 2017 IEEE SENSORS. IEEE, 2017, pp. 1-3.

[9] V. Mishra, N. Singh, U. Tiwari, and P. Kapur, "Fiber grating sensors in medicine: Current and emerging applications," Sensors and Actuators A: Physical, vol. 167, no. 2, pp. 279-290, 2011. 
[10] E. Al-Fakih, N. A. Abu Osman, and F. R. Mahamd Adikan, "The use of fiber bragg grating sensors in biomechanics and rehabilitation applications: the state-of-the-art and ongoing research topics," Sensors, vol. 12, no. 10, pp. 12890-12926, 2012.

[11] A. Beisenova, A. Issatayeva, I. Iordachita, W. Blanc, C. Molardi, and D. Tosi, "Distributed fiber optics 3d shape sensing by means of high scattering np-doped fibers simultaneous spatial multiplexing," Optics express, vol. 27, no. 16, pp. 22074-22 087, 2019.

[12] A. Issatayeva, A. Amantayeva, W. Blanc, D. Tosi, and C. Molardi, "Design and analysis of a fiber-optic sensing system for shape reconstruction of a minimally invasive surgical needle," Scientific reports, vol. 11, no. 1, pp. 1-12, 2021.

[13] K. R. Henken, J. Dankelman, J. J. van den Dobbelsteen, L. K. Cheng, and M. S. van der Heiden, "Error analysis of fbg-based shape sensors for medical needle tracking," IEEE/ASME Transactions on mechatronics, vol. 19, no. 5, pp. 1523-1531, 2013.

[14] S. Rusinkiewicz and M. Levoy, "Efficient variants of the icp algorithm," in Proceedings third international conference on 3-D digital imaging and modeling. IEEE, 2001, pp. 145-152.

[15] A. Dore, G. Smoljkic, E. Vander Poorten, M. Sette, J. Vander Sloten, and G.-Z. Yang, "Catheter navigation based on probabilistic fusion of electromagnetic tracking and physically-based simulation," in 2012 IEEE/RSJ International Conference on Intelligent Robots and Systems. IEEE, 2012, pp. 3806-3811.

[16] M. Azizian and R. Patel, "Data fusion for catheter tracking using kalman filtering: applications in robot-assisted catheter insertion," in Medical Imaging 2011: Visualization, Image-Guided Procedures, and Modeling, vol. 7964. International Society for Optics and Photonics, 2011, p. 796413.

[17] F. Alambeigi, S. A. Pedram, J. L. Speyer, J. Rosen, I. Iordachita, R. H. Taylor, and M. Armand, "Scade: Simultaneous sensor calibration and deformation estimation of fbg-equipped unmodeled continuum manipulators," IEEE Transactions on Robotics, 2019.

[18] R. Zhang, H. Liu, and J. Han, "Robust tracking of dexterous continuum robots: Fusing fbg shape sensing and stereo vision," in 2017 39th Annual International Conference of the IEEE Engineering in Medicine and Biology Society (EMBC). IEEE, 2017, pp. 925-928.

[19] S. Jäckle, V. García-Vázquez, F. von Haxthausen, T. Eixmann, M. M. Sieren, H. Schulz-Hildebrandt, G. Hüttmann, F. Ernst, M. Kleemann, and T. Paetz, " $3 \mathrm{~d}$ catheter guidance including shape sensing for endovascular navigation," in Medical Imaging 2020: Image-Guided Procedures, Robotic Interventions, and Modeling, vol. 11315. International Society for Optics and Photonics, 2020, p. 1131504.

[20] P.-L. Chang, A. Rolls, H. De Praetere, E. Vander Poorten, C. V. Riga, C. D. Bicknell, and D. Stoyanov, "Robust catheter and guidewire tracking using b-spline tube model and pixel-wise posteriors," IEEE Robotics and Automation Letters, vol. 1, no. 1, pp. 303-308, 2016.

[21] B. K. Horn, "Closed-form solution of absolute orientation using unit quaternions," Josa a, vol. 4, no. 4, pp. 629-642, 1987.

[22] M. G. Cox, "The numerical evaluation of b-splines," IMA Journal of Applied Mathematics, vol. 10, no. 2, pp. 134-149, 1972.

[23] A. Uneri, Y. Otake, A. Wang, G. Kleinszig, S. Vogt, A. J. Khanna, and J. Siewerdsen, "3d-2d registration for surgical guidance: effect of projection view angles on registration accuracy," Physics in Medicine \& Biology, vol. 59, no. 2, p. 271, 2013.

[24] S. Bisplinghoff, M. de la Fuente, M. Becker, and K. Radermacher, "Registration method for displaying electromagnetically tracked devices in fluoroscopic images," in 2010 Annual International Conference of the IEEE Engineering in Medicine and Biology. IEEE, 2010, pp. 37193722.

[25] A. F. Frangi, W. J. Niessen, K. L. Vincken, and M. A. Viergever, "Multiscale vessel enhancement filtering," in International conference on medical image computing and computer-assisted intervention. Springer, 1998, pp. 130-137.

[26] Z. Zhang, "A flexible new technique for camera calibration," IEEE Transactions on pattern analysis and machine intelligence, vol. 22, no. 11, pp. 1330-1334, 2000.

[27] A. Geiger, F. Moosmann, Ö. Car, and B. Schuster, "Automatic camera and range sensor calibration using a single shot," in 2012 IEEE International Conference on Robotics and Automation. IEEE, 2012, pp. 3936-3943.

[28] T.-C. Lee, R. L. Kashyap, and C.-N. Chu, "Building skeleton models via 3-d medial surface axis thinning algorithms," CVGIP: Graphical Models and Image Processing, vol. 56, no. 6, pp. 462-478, 1994.

[29] J. H. Lee, J. Kim, M. Kim, J. Hwang, Y. M. Hwang, J.-W. Kang, G.B. Nam, K.-J. Choi, and Y.-H. Kim, "Extremely low-frame-rate digital fluoroscopy in catheter ablation of atrial fibrillation: A comparison of 2 versus 4 frame rate," Medicine, vol. 96, no. 24, 2017. 